\title{
Monocyte activation in early onset rheumatoid arthritis
}

\author{
I Fujii, M Shingu, M Nobunaga
}

\begin{abstract}
Monocytes from peripheral blood and synovial fluid of patients with definite and classic rheumatoid arthritis spontaneously produced significantly greater amounts of prostaglandin $E_{2}\left(P_{G E}\right)$, leucotriene $B_{4}\left(L_{T B}\right)$, and interleukin-1 $\beta$ (IL-1 $\beta$ ) than samples of peripheral blood from normal controls. Peripheral blood monocytes from patients with rheumatoid arthritis produced significantly greater amounts of $\mathrm{PGE}_{2}$ than control samples when stimulated with lipopolysaccharide. There were no significant differences in the spontaneous release of superoxide or $\boldsymbol{N}$-acetyl- $\beta$ D-glucosaminidase by peripheral blood monocytes between patients and healthy controls. Both stimulated and unstimulated peripheral blood monocytes from patients with definite or classic rheumatoid arthritis produced significantly greater amounts of $\mathrm{PGE}_{2}$ than samples from normal controls. This was true, regardless of the stage of disease and the presence or absence of roentgenological joint abnormalities. Amounts of $\boldsymbol{N}$-acetyl- $\beta$-Dglucosaminidase released by peripheral blood monocytes from patients correlated positively with the erythrocyte sedimentation rate (ESR) and negatively with duration of disease. Amounts of IL-1 $\beta$ and $N$-acetyl- $\beta$-D-glucosaminidase released from the peripheral blood monocytes of patients who had had their disease for less than one year were significantly higher than those of normal controls. There were no significant correlations between the types of treatment and the amounts of $\mathrm{PGE}_{2}$, $\mathrm{LTB}_{4}, \mathrm{IL}-1 \beta$ or $\boldsymbol{N}$-acetyl- $\beta$-D-glucosaminidase released by peripheral blood monocytes in patients with rheumatoid arthritis.
\end{abstract}

The findings suggest that monocytes are activated in patients with rheumatoid arthritis both at the onset of disease and during its chronic phase, and that they produce large amounts of mediators which may have a role in the induction and extension of the inflammatory process which leads to tissue damage.

\footnotetext{
Department of Clinical Immunology, Medical Institute of Bioregulation, Kyushu University 69, Beppu, 874 Japan I Fujii M Shing

M Nobunaga

Correspondence to: Dr Fujii.

Accepted for publication 18 August 1989
}

Rheumatoid arthritis affects peripheral joints, and the destruction of joint structures, demonstrable on $x$ ray examination, is the most important feature for prognosis. The aetiology and pathogenesis of rheumatoid arthritis are poorly understood, but there are many indications that immune mechanisms may be involved. Macrophages are prominent in the accumulation of inflammatory cells in synovial tissue and fluid. The presence of increased concentrations, of HLA-DR antigens on cells from peripheral blood, synovial fluid, and synovium of patients with rheumatoid arthritis suggests that these cells are in an activated state. ${ }^{1}$ Changes in expression of certain surface antigens such as HLA-DR parallel the increased functional capacity of the cells. Thus cells originating from monocyte-macrophages are considered to have an important aetiological role in chronic inflammation ${ }^{2} 3$ by participating in the initiation and maintenance of the inflammatory process and subsequent tissue damage through the release of degrading lysosomal enzymes, ${ }^{4-6}$ superoxide anion $\left(\mathrm{O}_{2}^{-}\right),{ }^{7}$ the prostaglandin $\mathrm{E}_{2}\left(\mathrm{PGE}_{2}\right)^{89}$ and interleukin-1 (IL-1). ${ }^{10-12}$ The best characterised product of monocyte-macrophages is IL-1. It has been shown that IL-1 plays an important part in tissue damage by inducing a variety of functional changes of pathogenetic importance in other cells. ${ }^{47}$ 13-15 Stimulation of monocytes also leads to the release of lysosomal enzymes and activation of enzymes which produce mediators of inflammation. These include membrane bound nicotinamide-adenine dinucleotide phosphate oxidase (NADPH oxidase), which generates $\mathrm{O}_{2}^{-}$, and a 5-lipo-oxygenase, which catalyses the production of leucotrienes $\left(\mathrm{LTB}_{4}\right)$. Lysosomal proteinases and $\mathrm{O}_{2}^{-}$radicals are thought to be largely responsible for the resulting tissue injury found during an inflammatory reaction. Monocytes in rheumatoid arthritis have been shown to possess enhanced $F c$ receptor binding activity ${ }^{16}$ and enhanced metabolic and secretory activity. ${ }^{17}$ Monocytes in rheumatoid arthritis also produce increased amounts of $\mathrm{PGE}_{2}{ }^{18}$ and IL-1. 1920

The aim of this study was to determine whether the release of $\mathrm{PGE}_{2}, \mathrm{LTB}_{4}, \mathrm{IL}-1, \mathrm{O}_{2}^{-}$ and $N$-acetyl- $\beta$-D-glucosaminidase, a lysosomal proteinase, from monocytes is a specific marker of the early stage of rheumatoid arthritis and to correlate these mediators with inflammatory variables in patients with rheumatoid arthritis.

\section{Methods}

Forty four patients with rheumatoid arthritis (38 women, six men, mean age 55.7 years) satisfying the American Rheumatism Association (ARA) criteria $^{21}$ for classic or definite rheumatoid arthritis were investigated. The disease stage of joints in these patients was determined according to Steinbrocker et al. ${ }^{22} x$ Ray pictures of the joints were interpreted blind. The mean duration of rheumatoid arthritis was $7 \cdot 7$ years (range three months to $17 \cdot 3$ years).

Disease activity was estimated by the Lansbury activity index. ${ }^{23}$ Forty patients with definite and classic rheumatoid arthritis were 
receiving non-steroidal anti-inflammatory drugs (NSAIDs); 23 patients were being treated with steroids and seven with gold salts. The presence of vasculitis in patients with rheumatoid arthritis was evaluated either clinically or histologically, as previously described. ${ }^{24}$ Ten healthy female and six healthy male hospital employees, with a mean age of 40.6 years (range 23.8 to 57.4 years) served as controls.

Serum rheumatoid factor was measured by laser nephelometry (Behring) using a rheumatoid arthritis test kit (Hoechst) (normal range below $13 \mathrm{IU} / \mathrm{ml}$ ). Total haemolytic complement concentrations ( $\mathrm{CH} 50)$ were measured according to the method of Kent and Fife. ${ }^{25} \mathrm{C} 3$ and $\mathrm{C} 4$ were measured by single radial immunodiffusion using commercial kits (Hoechst).

\section{ISOLATION OF MONOCYTES}

Twenty millilitres of venous blood treated with heparin were collected, diluted with an equal volume of phosphate buffered saline (PBS) (0.01 $\mathrm{M}$ phosphate, $0.138 \mathrm{M} \mathrm{NaCl}, \mathrm{pH} 7 \cdot 4)$, and the mononuclear cells were isolated by Ficoll-Hypaque density gradient centrifugation (Pharmacia Fine Chemicals). Synovial fluid was aspirated from the knee joints of patients with rheumatoid arthritis into polystyrene tubes containing sodium heparin, diluted $1 / 1$ with PBS, and centrifuged at $200 \mathrm{~g}$. Cells were resuspended in PBS, layered on Ficoll-Hypaque, and centrifuged. Cells at the resulting interface were aspirated, washed twice in PBS, and suspended at a concentration of $2 \times 10^{6} / \mathrm{ml}$ in RPMI 1640 medium, supplemented with $100 \mathrm{U} / \mathrm{ml}$ penicillin, $100 \mu \mathrm{g} / \mathrm{ml}$ streptomycin, $0.25 \mathrm{ng} / \mathrm{ml}$ fungizone, $2 \mathrm{mmol} / \mathrm{ml} \mathrm{L}$-glutamine, and $10 \%$ heat inactivated fetal calf serum (FCS) (Gibco). These cells were then incubated in Petri dishes (Falcon, 3002) which had been coated with FCS and then incubated for one hour at $37^{\circ} \mathrm{C}$ in a $5 \%$ carbon dioxide incubator. Non-adherent cells were gently poured from the dishes and discarded. The dishes were rinsed with medium, and adherent cells were released into RPMI medium by mechanical agitation. The resultant cell suspension was centrifuged and the pellets were washed twice with medium. Cells were resuspended in 10\% FCS-RPMI 1640 at a concentration of $1 \times 10^{6} / \mathrm{ml}$ and constituted the monocyte preparation. The percentage of monocytes, as judged by Giemsa and esterase staining, varied between $95 \%$ and $98 \%$, and the viability, estimated by trypan blue dye exclusion, varied between $97 \%$ and $99 \%$. In some experiments monocytes were also suspended in Hanks's buffered salt solution (HBSS).

\section{CULTURE OF MONOCYTES}

Monocyte suspension $(0.5 \mathrm{ml})$ was mixed with $0.05 \mathrm{ml}$ of $50 \mu \mathrm{g} / \mathrm{ml}$ lipopolysaccharide (Sigma) as a stimulating agent, in polystyrene tubes (Falcon). Cells incubated with PBS instead of lipopolysaccharide served as unstimulated controls. Tubes were kept at $37^{\circ} \mathrm{C}$ in a $5 \%$ carbon dioxide incubator for 24 hours and then centrifuged at $400 \mathrm{~g}$ for 10 minutes. The supernatants were collected and frozen at $-80^{\circ} \mathrm{C}$ until use when they were assayed for $\mathrm{PGE}_{2}$, $\mathrm{LTB}_{4}, N$-acetyl- $\beta$-D-glucosaminidase, and IL$1 \beta$. As a stimulating agent, heat aggregated human IgG was used. This was prepared by heating native IgG (Cohn Fraction II, Sigma, $10 \mathrm{mg} / \mathrm{ml}$ ) at $37^{\circ} \mathrm{C}$ for 20 minutes, followed by centrifugation at $3000 \mathrm{rpm}$ for 20 minutes to remove large aggregates. The final concentration of heat aggregated human IgG was $500 \mu \mathrm{g} / \mathrm{ml}$.

A competitive binding radioimmunoassay was used for $\mathrm{PGE}_{2}$ using a commercial kit (Amersham). Leucotriene $B_{4}$ was also measured by radioimmunoassay using a commercial kit (Amersham).

$N$-acetyl- $\beta$-D-glucosaminidase was measured as described previously. ${ }^{26}$ Briefly, $0.2 \mathrm{ml}$ of sample, diluted $1 / 1$ with $0.1 \mathrm{ml}$ of $0.1 \mathrm{M}$ citrate

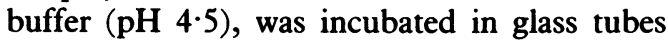
with $3.6 \mathrm{mM}$ paranitrophenyl-2-acetamido-2deoxy- $\beta$-D-glucopyranoside (Sigma) as substrate for four hours at $37^{\circ} \mathrm{C}$. The reaction was terminated with $0.3 \mathrm{ml}$ of $1 \mathrm{M}$ borate buffer (pH 9.8) and enzyme activity was determined colorimetrically at $405 \mathrm{~nm}$ as paranitrophenol was released from the substrate. Values were corrected for background levels in the medium.

$\mathrm{O}_{2}^{-}$generation by monocytes was measured as described previously. ${ }^{27}$ Monocytes $(0.2 \mathrm{ml})$ suspended in HBSS were plated in 96-well plates (Falcon), and ferricytochrome $c$ (horse heart, type III, Sigma) was added at the final concentration of $55 \mu \mathrm{mol} / 1$. In control wells $\mathrm{O}_{2}^{-}$ dismutase (SOD, Sigma $3000 \mathrm{U} / \mathrm{mg}, 20 \mu \mathrm{g} / \mathrm{ml}$ ) was added. Cells were then cultured for four hours at $37^{\circ} \mathrm{C}$. After incubation supernatants were obtained, diluted in distilled water, and reduction of ferricytochrome $c$ was measured by reading absorbance at $550 \mathrm{~nm}$ using a Beckman model 35 spectrophotometer. The amount of ferricytochrome $c$ reduction inhibited by SOD was calculated as described previously. ${ }^{28}$ Rates of ferricytochrome $c$ reduction were linear even at four hours.

IL-1 $\beta$ was measured by enzyme linked immunosorbent assay (ELISA) using a kit recently developed by Otsuka Pharmaceutical Co Ltd. ${ }^{29}$ Ninety six well-plates were coated overnight at room temperature with $0.1 \mathrm{ml}$ of antihuman mouse IL-1 $\beta$ monoclonal antibody ${ }^{30}$ diluted with PBS to a final concentration of 10 $\mu \mathrm{g} / \mathrm{ml}$. Plates were then washed three times with PBS. To avoid non-specific adsorption, $0.4 \mathrm{ml}$ of $1 \%$ bovine serum albumin (Sigma) was added to each well. Plates were kept at room temperature for four hours. After the blocking solution was removed each well was washed with $0.4 \mathrm{ml}$ of PBS and $0.05 \%$ Tween 20 . A solution of IL-1 $\beta$ containing $40 \mathrm{ng} / \mathrm{ml}$ of human recombinant IL-1 $\beta$ was serially diluted with PBS and the dilutions used as standards. ${ }^{31}$ Samples or serially diluted standards $(0.1 \mathrm{ml})$ were added to each well and incubated at $4^{\circ} \mathrm{C}$ overnight. Each well was washed three times with PBS after the samples or standards had been removed. Rabbit antihuman IL-1 $\beta$ polyclonal antibody $(0.1 \mathrm{ml})$ was added to each well and incubated at room temperature for two hours. Wells were washed three times with PBS. Horseradish peroxidase-conjugated antirabbit IgG $(0 \cdot 1 \mathrm{ml})$ was added to each well. 
After incubation for two hours at room temperature the wells were washed with PBS and $0.1 \mathrm{ml}$ of substrate solution $(0.02 \%$ hydrogen peroxide, $1 \mathrm{mg} / \mathrm{ml} 0$-phenylenediamine dihydrochloride in $0.1 \mathrm{M}$ phosphate buffer, $\mathrm{pH} 5.5$ ) was added. Plates were kept at room temperature for 10-15 minutes and the reaction was stopped by adding $0.1 \mathrm{ml}$ of $1 \mathrm{~N} \mathrm{H}_{2} \mathrm{SO}_{4}$ solution. Absorbance was measured at $492 \mathrm{~nm}$ using an ELISA reader (Dainippon-Sei-yaku Co Ltd). This ELISA assay detects $15 \cdot 6-500 \mathrm{pg} / \mathrm{ml}$ of IL-1 $\beta$.

Levels of significance were calculated by Student's $t$ test.

\section{Results}

Peripheral blood monocytes or synovial fluid monocytes from patients with classic or definite rheumatoid arthritis spontaneously produced significantly greater amounts of $\mathrm{PGE}_{2}, \mathrm{LTB}_{4}$, and IL-1 $\beta$ than peripheral blood monocytes from normal controls. Peripheral blood monocytes from patients with rheumatoid arthritis produced significantly greater amounts of $\mathrm{PGE}_{2}$ compared with normal controls when stimulated with lipopolysaccharide. There were no significant differences in the spontaneous release of $N$ acetyl- $\beta$-D-glucosaminidase or $\mathrm{O}_{2}^{-}$by peripheral blood monocytes between patients with rheumatoid arthritis and healthy controls (table 1). When the stimulating effects of lipopolysaccharide and heat aggregated human IgG on $\mathrm{PGE}_{2}$ release by peripheral blood monocytes in rheumatoid arthritis were compared, both lipopolysaccharide and heat aggregated human IgG stimulated $\mathrm{PGE}_{2}$ production by peripheral blood monocytes in rheumatoid arthritis to the same extent (data not shown).

Both stimulated and unstimulated peripheral blood monocytes from patients with definite or classic rheumatoid arthritis produced significantly higher amounts of $\mathrm{PGE}_{2}$ than normal peripheral blood monocytes. Leucotriene $\mathrm{B}_{4}$ production by peripheral blood monocytes from patients with classic rheumatoid arthritis was significantly higher than that by normal peripheral blood monocytes. Production of IL-1 $\beta$, $\mathrm{O}_{2}^{-}$, or $N$-acetyl- $\beta$-D-glucosaminidase by peripheral blood monocytes from patients with any of these diagnostic classifications was within the normal range. Patients with definite and classic rheumatoid arthritis were classified by the stage of their joints determined by $x$ ray, and amounts of mediators released from peripheral blood monocytes were compared with those released from normal peripheral blood monocytes. Peripheral blood monocytes from patients with rheumatoid arthritis with no joint abnormalities spontaneously produced significantly higher amounts of $\mathrm{PGE}_{2}$ and IL- $1 \beta$ than normal peripheral blood monocytes. Similarly, peripheral blood monocytes from patients with rheumatoid arthritis with stages I, III, IV changes spontaneously produced significantly higher amounts of $\mathrm{PGE}_{2}$ than normal peripheral blood monocytes. Peripheral blood monocytes stimulated with lipopolysaccharide from patients with rheumatoid arthritis with stages II, III, or IV changes released significantly higher amounts of $\mathrm{PGE}_{2}$ than normal peripheral blood monocytes. Leucotriene $B_{4}$ production by peripheral blood monocytes from patients with rheumatoid arthritis with stages II, III, or IV changes was significantly higher than that in normal peripheral blood monocytes. Only peripheral blood monocytes from patients with rheumatoid arthritis and stage II changes showed significantly increased $\mathrm{O}_{2}^{-}$release compared with normal peripheral blood monocytes; in the other groups the values were not increased. Release of $N$-acetyl- $\beta$-D-glucosaminidase by monocytes from patients with rheumatoid arthritis with stages I and III changes was significantly increased compared with that of normal peripheral blood monocytes (table 2).

The amounts of $N$-acetyl- $\beta$-D-glucosaminidase released by peripheral blood monocytes from patients with rheumatoid arthritis correlated positively with ESR and negatively with duration of disease. The amount of $N$-acetyl- $\beta$-D-glucosaminidase released, however, did not correlate with activity index (table 3 ). There were no significant correlations between the amounts of $\mathrm{PGE}_{2}, \mathrm{LTB}_{4}, \mathrm{O}_{2}^{-}$or IL- $1 \beta$ and ESR, activity index, or duration of disease. There were no differences in the amounts of mediators released by peripheral blood monocytes in rheumatoid arthritis in the presence or absence of vasculitis (data not shown). When patients with rheumatoid arthritis were classified according to disease duration of less or more than one year and the amounts of mediators released by unstimulated peripheral blood monocytes were compared with those of healthy controls, the amounts of IL-1 $\beta$ and $N$-acetyl- $\beta$-D-glucosaminidase produced were significantly higher in patients with rheumatoid arthritis with disease duration less than one year. On the other hand, the production of $\mathrm{PGE}_{2}$ and $\mathrm{LTB}_{4}$ was not increased in patients with rheumatoid arthritis with disease duration of less than one year, but was significantly increased in patients with rheumatoid arthritis with disease duration of more than one year (table 4).

Table 1: Amounts of mediators (mean $(S D)$ ) released by peripheral or synovial fluid monocytes from patients and normal controls

\begin{tabular}{|c|c|c|c|c|c|c|c|c|}
\hline Mediators released & $\begin{array}{l}\text { Periph } \\
\text { (norma }\end{array}$ & $\begin{array}{l}\text { blood } \\
\text { ontrols) }\end{array}$ & ocytes & $\begin{array}{l}\text { Perip) } \\
\text { (rheun }\end{array}$ & $\begin{array}{l}\text { l blood } n \\
\text { id arthrit }\end{array}$ & ocytes & $\begin{array}{l}\text { Synor } \\
\text { (rheur }\end{array}$ & $\begin{array}{l}\text { l fluid monocytes } \\
\text { utoid arthritis) }\end{array}$ \\
\hline $\begin{array}{l}\mathrm{PGE}_{2}^{*}\left(\mathrm{pg} / 10^{6} \text { cells }\right) \text { unstimulated } \\
\mathrm{LPS}^{*} \text { stimulated } \\
\mathrm{LTB}_{4}\left(\mathrm{pg} / 10^{6} \text { cells }\right) \text { unstimulated } \\
\mathrm{IL}^{-1 \beta^{*}}\left(\mathrm{ng} / 10^{6} \text { cells }\right) \text { unstimulated } \\
\mathrm{NAG}^{*}(\mu \mathrm{mol}) \text { unstimulated } \\
\mathrm{O}_{2}^{-}(\mathrm{nmol}) \text { unstimulated }\end{array}$ & $\begin{array}{l}2741 \\
4898 \\
91 \cdot 2 \\
56 \cdot 4 \\
314 \cdot 5 \\
0 \cdot 99\end{array}$ & $\begin{array}{l}(1649) \\
(3671) \\
(48 \cdot 7) \\
(58 \cdot 5) \\
(16 \cdot 6) \\
(0 \cdot 25)\end{array}$ & $\begin{array}{l}(n=16) \\
(n=16) \\
(n=15) \\
(n=8) \\
(n=6) \\
(n=9)\end{array}$ & $\begin{array}{l}5935 \\
9830 \\
146 \cdot 4 \\
122 \cdot 9 \\
331 \cdot 9 \\
1 \cdot 16\end{array}$ & $\begin{array}{l}(3748) \ddagger \\
(5873) \ddagger \\
(58 \cdot 4) \ddagger \\
(79 \cdot 3)^{*} \\
(24 \cdot 5) \\
(0 \cdot 49)\end{array}$ & $\begin{array}{l}(n=44) \\
(n=44) \\
(n=43) \\
(n=24) \\
(n=35) \\
(n=20)\end{array}$ & $\begin{array}{l}3425 \\
113 \cdot 8 \\
47 \cdot 9 \\
-\end{array}$ & $\begin{array}{ll}(2540) & (n=12)+t \\
& \\
(44 \cdot 4) & (n=11) \\
(44 \cdot 7) & (n=8) \uparrow\end{array}$ \\
\hline
\end{tabular}


Table 5 shows that steroids, gold salts, or NSAIDs did not affect $\mathrm{PGE}_{2}$ production by peripheral blood monocytes in patients with rheumatoid arthritis. Similarly, these drugs did not influence the production of $\mathrm{LTB}_{4}, \mathrm{IL}-1 \beta$, or $N$-acetyl- $\beta$-D-glucosaminidase by peripheral blood monocytes. $\mathrm{O}_{2}^{-}$release by peripheral blood monocytes obtained from patients with rheumatoid arthritis taking gold salts was significantly higher than in patients not taking gold salts; other agents did not affect $\mathrm{O}_{2}^{-}$ release (table 5).

Table 2: Amounts (mean $(S D)$ ) of mediators released by peripheral blood monocytes from patients in relation to diagnostic criteria and stage at $x$ ray

\begin{tabular}{|c|c|c|c|c|c|c|}
\hline & \multicolumn{2}{|l|}{$P G E_{2}^{*}(p g)$} & \multirow[t]{2}{*}{$L T B_{4}^{*}(p g)$} & \multirow[t]{2}{*}{$I L-l \beta(n g / m l)$} & \multirow[t]{2}{*}{$\mathrm{O}_{2}^{-}(\mathrm{nmol})$} & \multirow[t]{2}{*}{$N A G^{*}(\mu \mathrm{mol} / \mathrm{l})$} \\
\hline & $L P S^{*}(-)$ & $\operatorname{LPS}(+)$ & & & & \\
\hline Normal controls & $\begin{array}{l}2741(1649) \\
(n=16)\end{array}$ & $\begin{array}{c}4898(3671) \\
(\mathrm{n}=16)\end{array}$ & $\begin{array}{l}91 \cdot 2(48 \cdot 7) \\
(n=15)\end{array}$ & $\begin{array}{c}56 \cdot 4 \\
(\mathrm{n}=8)\end{array}(58 \cdot 5)$ & $\begin{array}{l}0.99(0.25) \\
(\mathrm{n}=9)\end{array}$ & $\begin{array}{l}314 \cdot 5(16 \cdot 6) \\
(n=6)\end{array}$ \\
\hline Definite rheumatoid arthritis & $\begin{array}{l}6206(4705) \dagger \\
(n=16)\end{array}$ & $\begin{array}{l}9945(5961)^{*} \\
(\mathbf{n}=16)\end{array}$ & $\begin{array}{l}121 \cdot 1(67 \cdot 6) \\
(\mathrm{n}=19)\end{array}$ & $\begin{array}{l}107 \cdot 3(72 \cdot 5) \\
(\mathbf{n}=11)\end{array}$ & $\begin{array}{l}0.73(0.43) \\
(n=7)\end{array}$ & $\begin{array}{l}332 \cdot 5(19 \cdot 5) \\
(\mathbf{n}=15)\end{array}$ \\
\hline Classic rheumatoid arthritis & $\begin{array}{l}5638(2722) \mathrm{tt} \\
(\mathrm{n}=28)\end{array}$ & $\begin{array}{l}10299(6378) \dagger \\
(n=28)\end{array}$ & $\begin{array}{l}164 \cdot 5(56 \cdot 4)+t \\
(n=24)\end{array}$ & $\begin{array}{l}124 \cdot 5 \\
(n=13)\end{array}$ & $\begin{array}{l}1 \cdot 24(0.52) \\
(n=13)\end{array}$ & $\begin{array}{l}330 \cdot 2(27 \cdot 4) \\
(n=20)\end{array}$ \\
\hline Stage 0 & $\begin{array}{l}6438(2063) \dagger \\
(n=2)\end{array}$ & $\begin{array}{l}8000(1000) \\
(n=2)\end{array}$ & $\begin{array}{l}119 \cdot 1(68 \cdot 0) \\
(n=4)\end{array}$ & $\begin{array}{l}150 \cdot 0 \\
(\mathrm{n}=3)\end{array}(86 \cdot 8)^{*}$ & $\begin{array}{l}0.76 \\
(n=1)\end{array}$ & $\begin{array}{l}320 \cdot 3(18 \cdot 5) \\
(n=3)\end{array}$ \\
\hline Stage I & $\begin{array}{l}4338(2448)^{*} \\
(n=14)\end{array}$ & $\begin{array}{l}9194(7438) \\
(n=14)\end{array}$ & $\begin{array}{l}141 \cdot 9(97 \cdot 5) \\
(n=8)\end{array}$ & $\begin{array}{l}112 \cdot 0 \\
(n=5)\end{array}(84 \cdot 4)$ & $\begin{array}{l}1 \cdot 01(0.58) \\
(n=6)\end{array}$ & $\begin{array}{c}343 \cdot 1 \\
(n=8)\end{array}(18 \cdot 5) \dagger$ \\
\hline Stage II & $\begin{array}{l}7036(6074) \\
(n=8)\end{array}$ & $\begin{array}{l}11251 \\
(\mathbf{n}=8)\end{array}(5630) \dagger$ & $\begin{array}{l}145 \cdot 6(59 \cdot 0) \dagger \\
(n=15)\end{array}$ & $\begin{array}{l}123 \cdot 6 \\
(\mathrm{n}=8)\end{array}$ & $\begin{array}{l}1 \cdot 38(0 \cdot 37)^{*} \\
(\mathbf{n}=6)\end{array}$ & $\begin{array}{l}329 \cdot 9(29 \cdot 3) \\
(\mathbf{n}=9)\end{array}$ \\
\hline Stage III & $\begin{array}{l}7016(2035) \dagger \\
(\mathbf{n}=8)\end{array}$ & $\begin{array}{l}11062(4329) \ddagger \\
(n=8)\end{array}$ & $\begin{array}{l}165 \cdot 3(77 \cdot 4) \dagger \\
(n=7)\end{array}$ & $\begin{array}{l}135 \cdot 7(111 \cdot 1) \\
(n=3)\end{array}$ & $\begin{array}{l}0 \cdot 84(0 \cdot 31) \\
(\mathrm{n}=3)\end{array}$ & $\begin{array}{l}338 \cdot 3(11 \cdot 0) \dagger \\
(n=8)\end{array}$ \\
\hline Stage IV & $\begin{array}{l}5865(3330) \ddagger \\
(\mathrm{n}=12)\end{array}$ & $\begin{array}{l}9427(4667)^{+} \\
(n=12)\end{array}$ & $\begin{array}{l}152 \cdot 5(56 \cdot 8) \dagger \\
(n=9)\end{array}$ & $\begin{array}{l}108 \cdot 8 \\
(\mathbf{n}=5)\end{array}$ & $\begin{array}{l}1 \cdot 22(0 \cdot 72) \\
(n=4)\end{array}$ & $\begin{array}{l}319 \cdot 6(33 \cdot 4) \\
(n=7)\end{array}$ \\
\hline
\end{tabular}

Patients were categorised according to diagnostic criteria, and patients with definite or classic rheumatoid arthritis were classified by stage at $x$ ray. ${ }^{*} \mathrm{p}<0.05 ; \mathrm{t} \mathbf{p}<0.02 ; \neq \mathrm{p}<0.01 \mathrm{tp}<0.001$ (v normal).

*For abbreviations see table 1 .

Table 3: Relation between amounts of mediators released from peripheral blood monocytes and clinical variables in patients

\begin{tabular}{|c|c|c|c|c|c|}
\hline Variables & $P G E_{2}{ }^{*}$ & $\mathrm{LTB}_{4}{ }^{*}$ & $N A G^{*}$ & $\mathrm{O}_{2}^{-}$ & $I L-I \beta^{*}$ \\
\hline ESR & $\begin{array}{l}r=0 \cdot 081 \\
n=44\end{array}$ & $\begin{array}{l}r=-0.02 \\
n=43\end{array}$ & $\begin{array}{l}r=0 \cdot 406^{*} \\
n=35\end{array}$ & $\begin{array}{l}r=0 \cdot 085 \\
n=20\end{array}$ & $\begin{array}{l}r=-0 \cdot 181 \\
n=24\end{array}$ \\
\hline Serum rheumatoid factor & $\begin{array}{l}r=-0.077 \\
n=44\end{array}$ & $\begin{array}{l}r=0 \cdot 082 \\
n=43\end{array}$ & - & & $\begin{array}{l}r=0 \cdot 182 \\
n=24\end{array}$ \\
\hline Activity index & $\begin{array}{l}r=0.008 \\
n=38\end{array}$ & $r=0.012$ & $\begin{array}{l}r=0.064 \\
n=22\end{array}$ & $\begin{array}{l}r=0 \cdot 226 \\
n=16\end{array}$ & $\begin{array}{l}r=-0 \cdot 183 \\
n=21\end{array}$ \\
\hline Duration of disease & $\begin{array}{l}r=0 \cdot 078 \\
n=44\end{array}$ & $\begin{array}{l}r=0 \cdot 171 \\
n=43\end{array}$ & $\begin{array}{l}r=-0.384 \\
n=34\end{array}$ & $\begin{array}{l}\mathrm{r}=-0 \cdot 161 \\
\mathrm{n}=20\end{array}$ & $\begin{array}{l}\mathrm{r}=-0.036 \\
\mathrm{n}=22\end{array}$ \\
\hline
\end{tabular}

Data are expressed as correlation coefficients ( $\mathrm{r}$ values). Patients include those with both definite and classic rheumatoid arthritis. ${ }^{*} \mathrm{p}<0 \cdot 05$.

For abbreviations see table 1 .

Table 4: Comparison of mediators released by unstimulated peripheral blood monocytes from normal controls and from patients with disease duration of greater or less than one year

\begin{tabular}{|c|c|c|c|c|c|c|c|c|c|}
\hline \multirow[t]{2}{*}{ Mediators } & \multicolumn{3}{|c|}{ Normal controls } & \multicolumn{6}{|c|}{ Disease duration } \\
\hline & & & & $<1$ year & & & $>1$ yed & & \\
\hline $\begin{array}{l}\mathrm{PGE}_{2}^{*}(\mathrm{pg}) \\
\mathrm{LTB}_{4}^{*}(\mathrm{pg}) \\
\mathrm{IL}^{*}-1 \beta^{*}(\mathrm{ng} / \mathrm{ml}) \\
\mathrm{O}_{2}^{-}(\mathrm{nmol}) \\
\mathrm{NAG}^{*}(\mu \mathrm{mol} / \mathrm{l})\end{array}$ & $\begin{array}{l}2741 \\
91 \cdot 2 \\
56 \cdot 4 \\
0 \cdot 99 \\
314 \cdot 5\end{array}$ & $\begin{array}{l}(1649) \\
(48 \cdot 7) \\
(58 \cdot 5) \\
(0 \cdot 25) \\
(16 \cdot 6)\end{array}$ & $\begin{array}{l}(\mathbf{n}=16) \\
(\mathbf{n}=15) \\
(\mathbf{n}=8) \\
(\mathbf{n}=9) \\
(\mathbf{n}=6)\end{array}$ & $\begin{array}{l}4688 \\
117 \cdot 1 \\
133 \cdot 8 \\
0 \cdot 81 \\
335 \cdot 2\end{array}$ & $\begin{array}{l}(3714) \\
(66 \cdot 1) \\
(63 \cdot 2)^{*} \\
(0 \cdot 38) \\
(16 \cdot 6)^{*}\end{array}$ & $\begin{array}{l}(\mathbf{n}=10) \\
(\mathbf{n}=11) \\
(\mathbf{n}=8) \\
(\mathbf{n}=5) \\
(\mathbf{n}=11)\end{array}$ & $\begin{array}{l}6247 \\
156 \cdot 5 \\
117 \cdot 5 \\
1 \cdot 27 \\
331 \cdot 3\end{array}$ & $\begin{array}{l}(3738) \ddagger \\
(67 \cdot 3) \dagger \\
(87 \cdot 6) \\
(0 \cdot 47) \\
(24 \cdot 6)\end{array}$ & $\begin{array}{l}(n=34) \\
(n=32) \\
(n=16) \\
(n=15) \\
(n=24)\end{array}$ \\
\hline
\end{tabular}

${ }^{*} \mathrm{p}<0.05 ; \mathrm{tp}<0.01 ; \neq \mathrm{p}<0.001$ ( $v$ normal).
${ }_{\text {*For abbreviations see table } 1 \text {. }}$.

Table 5: Effect of treatment regimen on amounts of mediators released by peripheral blood monocytes from patients

\begin{tabular}{|c|c|c|c|c|c|}
\hline Treatment & $P G E_{2}(p g)$ & $L T B_{4}(p g)$ & $\begin{array}{l}I L-1 \beta \\
(\mathbf{n g} / \mathbf{m l})\end{array}$ & $\begin{array}{l}\text { NAG } \\
(\mu \mathrm{mol} / \mathrm{l})\end{array}$ & $\mathrm{O}_{2}^{-} \quad(\mathrm{nmol})$ \\
\hline $\begin{array}{l}\text { Steroid (+) } \\
\text { Steroid (-) }\end{array}$ & $\begin{array}{l}6304(2952) \\
(n=23) \\
5620(4345) \\
(n=21)\end{array}$ & $\begin{array}{l}167 \cdot 9(89 \cdot 3) \\
(n=24) \\
145 \cdot 1(73 \cdot 6) \\
(n=19)\end{array}$ & $\begin{array}{l}111 \cdot 0(82 \cdot 8) \\
(n=15) \\
142 \cdot 8(73 \cdot 2) \\
(n=9)\end{array}$ & $\begin{array}{l}329 \cdot 9(27 \cdot 6) \\
(n=19) \\
335 \cdot 8(21 \cdot 7) \\
(n=16)\end{array}$ & $\begin{array}{l}1 \cdot 35(0 \cdot 47) \\
(n=10) \\
0 \cdot 90(0 \cdot 48) \\
(n=10)\end{array}$ \\
\hline $\begin{array}{l}\text { Gold (+) } \\
\text { Steroid (-) }\end{array}$ & $\begin{array}{l}5938(3196) \\
(n=4)\end{array}$ & $\begin{array}{l}118 \cdot 9(71 \cdot 7) \\
(n=7)\end{array}$ & $\begin{array}{l}89 \cdot 0(72 \cdot 5) \\
(\mathbf{n}=5)\end{array}$ & $\begin{array}{l}345 \cdot 5(26 \cdot 5) \\
(n=4)\end{array}$ & $\begin{array}{l}1.69(0 \cdot 12)^{t} \\
(n=3)\end{array}$ \\
\hline $\begin{array}{l}\text { Gold (-) } \\
\text { Steroid (-) } \\
\text { NSAIDs (+) }\end{array}$ & $\begin{array}{l}5563(4471) \\
(n=24) \\
6178(3724) \\
(n=40)\end{array}$ & $\begin{array}{l}143 \cdot 7(67 \cdot 7) \\
(n=15) \\
143 \cdot 2(69 \cdot 0) \\
(n=40)\end{array}$ & $\begin{array}{l}138 \cdot 6(80 \cdot 3) \\
(n=7) \\
119 \cdot 6(79 \cdot 3) \\
(n=23)\end{array}$ & $\begin{array}{l}334 \cdot 4(20 \cdot 4) \\
(n=13) \\
332 \cdot 7(24 \cdot 4) \\
(n=29)\end{array}$ & $\begin{array}{l}0 \cdot 83(0 \cdot 45) \\
(n=9) \\
1 \cdot 12(0 \cdot 50) \\
(n=15)\end{array}$ \\
\hline NSAIDs (-) & $\begin{array}{l}4963(3884) \\
(n=4)\end{array}$ & $\begin{array}{l}189 \cdot 6(50 \cdot 3) \\
(n=3)\end{array}$ & $\begin{array}{l}200 \cdot 0 \\
(n=1)\end{array}$ & $\begin{array}{l}328 \cdot 2(27 \cdot 5) \\
(n=6)\end{array}$ & $\begin{array}{l}1 \cdot 14(0 \cdot 61) \\
(n=5)\end{array}$ \\
\hline
\end{tabular}

Data are expressed as mean (SD), ${ }^{*} \mathrm{p}<0.05 v$ gold $(-)$ steroid $(-)$. 


\section{Discussion}

The data obtained suggest that peripheral blood monocytes from patients with rheumatoid arthritis spontaneously produce increased amounts of $\mathrm{PGE}_{2}, \mathrm{LTB}_{4}$, and IL-1 $\beta$ compared with normal peripheral blood monocytes. There was no increase in the amounts of $N$-acetyl- $\beta$-Dglucosaminidase and $\mathrm{O}_{2}^{-}$released by peripheral blood monocytes in rheumatoid arthritis. Interestingly, synovial fluid monocytes produced smaller amounts of $\mathrm{PGE}_{2}$ and IL-1 $\beta$ than peripheral blood monocytes from patients with rheumatoid arthritis. The reason for this is not clear. Prostaglandin $E_{2}$ released by peripheral blood monocytes from patients with rheumatoid arthritis and normal controls was significantly stimulated by the addition of lipopolysaccharide. Prostaglandin $\mathrm{E}_{2}$ released by peripheral blood monocytes in rheumatoid arthritis was also stimulated by heat aggregated human IgG, used as a model of an immune complex. Peripheral blood monocytes from patients with definite or classic rheumatoid arthritis without bone erosion (stage 0)-that is, early onset rheumatoid arthritis-produced significantly higher amounts of $\mathrm{PGE}_{2}$ and IL-1 $\beta$ than controls. Finally, peripheral blood monocytes from patients with rheumatoid arthritis with progressive bone lesions (stages II, III, or IV) also showed increased release of $\mathrm{PGE}_{2}, \mathrm{LTB}_{4}, \mathrm{O}_{2}^{-}$ and $N$-acetyl- $\beta$-D-glucosaminidase. These findings suggest that increased production of $\mathrm{PGE}_{2}$ and IL-1 $\beta$ by peripheral blood monocytes in rheumatoid arthritis is not only one of the early activation phenomena in monocytes in rheumatoid arthritis, but is also a sign of activation in the later stages of the disease. On the other hand, production of $\mathrm{LTB}_{4}, \mathrm{O}_{2}^{-}$, and $N$-acetyl$\beta$-D-glucosaminidase by peripheral blood monocytes was not increased in the early stage of rheumatoid arthritis, but was increased in the chronic and progressive stages. Furthermore, the amounts of $\mathrm{PGE}_{2}, \mathrm{LTB}_{4}, \mathrm{O}_{2}^{-}$or IL- $1 \beta$ spontaneously released by peripheral blood monocytes in rheumatoid arthritis did not correlate with ESR, serum rheumatoid factor concentrations, activity index or duration of disease. Only $N$-acetyl- $\beta$-D-glucosaminidase showed a positive correlation with ESR and a negative correlation with duration of disease.

Enhanced $\mathrm{PGE}_{2}$ release from monocytes was found both in the early stages of untreated rheumatoid arthritis and in patients receiving prolonged treatment with NSAIDs or disease modifying antirheumatic drugs. ${ }^{18}$ No correlation, however, between $\mathrm{PGE}_{2}$ released from monocytes in rheumatoid arthritis and systemic disease activity was observed. Enhanced $\mathrm{PGE}_{2}$ release was not specific for patients with rheumatoid arthritis as monocytes from patients with systemic lupus erythematosus also showed enhanced $\mathrm{PGE}_{2}$ release. ${ }^{18}$ Our data are similar to those previously described which have suggested that activation of cyclo-oxygenase occurs in patients with rheumatoid arthritis. A pathogenic role for $\mathrm{PGE}_{2}$ on joint tissue damage has been reported previously. ${ }^{32}$ Our data clearly show that $\mathrm{PGE}_{2}$ production is increased both at early and late stages of rheumatoid arthritis. In an animal model of arthritis in rats it was shown that induction and progression of arthritis was associated with both systemic (splenic, peritoneal) and local (synovial) macrophage activation, including enhanced production of $\mathrm{PGE}_{2}$ and IL-1 and increased Ia antigen expression. ${ }^{33}$ Production of another product of arachidonic acid, $\mathrm{LTB}_{4}$, is mediated by 5-lipo-oxygenase. Leucotriene $\mathrm{B}_{4}$ has potent chemotactic and degranulating actions, produces vascular permeability, and is reported to have a pro-inflammatory role in the arthritic joint. ${ }^{34}$ Our data indicate that peripheral blood monocytes in rheumatoid arthritis produce increased amounts of $\mathrm{LTB}_{4}$, but synovial fluid monocytes do not.

It has been shown that peripheral blood monocytes in rheumatoid arthritis release significantly greater amounts of IL-1 than normal peripheral blood monocytes, both stimulated and unstimulated, and that IL-1 generation by peripheral blood monocytes in rheumatoid arthritis is temporally linked to an early event in the onset or exacerbation of rheumatoid arthritis. ${ }^{20}$ Our data showed that IL-1 production was increased in an early stage of rheumatoid arthritis, but did not correlate with disease activity. The amounts of mediators released by unstimulated peripheral blood monocytes in rheumatoid arthritis were compared in patients with disease duration of less than one year and those with disease duration of more than one year. The data presented imply that the production of IL- $1 \beta$ and $N$-acetyl- $\beta$-Dglucosaminidase, but not $\mathrm{PGE}_{2}$ and $\mathrm{LTB}_{4}$, by unstimulated peripheral blood monocytes in rheumatoid arthritis is activated relatively early in rheumatoid arthritis and that in the chronic stage of the disease $\mathrm{PGE}_{2}$ and $\mathrm{LTB}_{4}$ production is activated but not that of IL- $1 \beta$ and $N$-acetyl$\beta$-D-glucosaminidase. Synovial fluid monocytes in rheumatoid arthritis have previously been shown to produce greater amounts of IL-1 than normal peripheral blood monocytes and comparable amounts of IL-1 with peripheral blood monocytes in rheumatoid arthritis. ${ }^{19}$ This contrasts with our finding that synovial fluid monocytes in rheumatoid arthritis produce significantly lower IL- $1 \beta$ than peripheral blood monocytes in rheumatoid arthritis. It has been also suggested that peripheral blood monocytes in rheumatoid arthritis, both stimulated and unstimulated, produce significantly lower IL-1 than normal peripheral blood monocytes. ${ }^{35}$

We used an ELISA to measure IL-1 $\beta$, whereas the previous group used an IL-1 dependent cell line to measure IL-1 activity. This difference in assay procedure might have influenced the results. It has been shown that IL-1 modulates functional properties of joint tissue cells such as synovial cells and chondrocytes. These modulating effects of IL-1 include stimulation of fibroblasts and synovial cells to proliferate, to secrete collagenase, and to produce collagen, $\mathrm{PGE}_{2}$, and lysosomal enzymes. Interleukin-1 also stimulates chondrocytes to produce $\mathrm{PGE}_{2}$, proteases, and other enzymes and inhibits proteoglycan synthesis.

Muirden $^{36}$ and others ${ }^{37}$ found that the synovium of patients with rheumatoid arthritis contained increased acid hydrolase activities. A recent report provides evidence that IL-1 stimu- 
lates the $N$-acetyl- $\beta$-D-glucosaminidase activity of synovial cells. ${ }^{15}$ At persistent or transient $\mathrm{pH}$ values low enough to promote clinically important activity of certain enzymes, such as might occur in the protected areas between synovium and cartilage, ${ }^{38} \mathrm{~N}$-acetyl- $\beta$-D-glucosaminidase may have an important role in the lysis of connective tissue components or activation of latent neutral proteases such as plasminogen activator. ${ }^{39}$

It has been suggested that rheumatoid monocyte metabolic activity, as measured by hexose monophosphate shunt activity, is stimulated compared with that in normal monocytes. Hurst et al showed that there was no significant difference in unstimulated rates of monocyte $\mathrm{O}_{2}^{-}$production between normal controls and patients with rheumatoid arthritis receiving NSAIDs alone, and patients with rheumatoid arthritis receiving second line treatment. ${ }^{40}$ This finding is very similar to our results.

Most experiments on monocyte $\mathrm{O}_{2}^{-}$generation have been done using an incubation period of one hour; ours were performed using an incubation period of four hours. It has been suggested that the differentiation of human monocytes into macrophages in vitro is accompanied by an apparent reduction in the capacity to produce $\mathrm{O}_{2}^{-}$and hydrogen peroxide, and that the rates of production of $\mathrm{O}_{2}^{-}$after three days in culture decreased to half of that initially cultured. ${ }^{41}$ These findings suggest that a four hour culture does not cause clinically important monocyte differentiation and a large decrease of $\mathrm{O}_{2}^{-}$production.

It has been implied that monocyte metabolic activity, in vitro and in vivo, is modulated by NSAIDs or disease modifying agents. For example, piroxicam, which inhibits cyclooxygenase, inhibits $\mathrm{O}_{2}^{-}$production mediated by $\mathrm{N}$-formyl-methionyl-leucyl-phenylalanine but not $\mathrm{LTB}_{4}$ production by monocytes. ${ }^{42}$ Successful treatment with $D$-penicillamine or gold compounds is associated with a significant increase in monocyte $\mathrm{O}_{2}^{-}$generation. ${ }^{43}$ It has also been shown that gold compounds inhibit IL-1 production by monocytes. ${ }^{44}$ Dexamethasone, a potent phospholipase $A_{2}$ inhibitor, was shown to inhibit IL-1 production by human monocytes. ${ }^{45}$ Finally, auranofin, but not gold salts, inhibited $\mathrm{LTB}_{4}$ production of normal peripheral blood monocytes. These observations strongly suggest that therapeutic agents in patients with rheumatoid arthritis modify the rates of production of various mediators by monocytes. When the patients were grouped into several categories according to the therapeutic agents they were receiving in our study and the rates of mediators released by peripheral blood monocytes were compared, there were no significant differences among the groups in the rates of PGE $2, \mathrm{LTB}_{4}, \mathrm{IL}-1 \beta$, and $N$-acetyl- $\beta$-Dglucosaminidase released by peripheral blood monocytes in rheumatoid arthritis. $\mathrm{O}_{2}^{-}$released by peripheral blood monocytes from patients receiving gold salts, however, was significantly greater than that of peripheral blood monocytes from patients not receiving gold salts. This finding agreed with the findings of the previous report. ${ }^{43}$
The data presented here suggest that monocytes are activated in patients with rheumatoid arthritis and that they produce increased amounts of mediators which may have a role in the induction and extension of the inflammatory process. The mechanisms by which monocytes are metabolically activated in patients with rheumatoid arthritis are not yet fully understood, but immune complexes and activated complement products may be responsible for monocyte activation. Monocytes from patients with rheumatoid arthritis are known to have increased numbers of $\mathrm{Fc}$ receptors ${ }^{16}$ and this may also furnish a mechanism for monocyte activation in these patients.

We thank Miss E Kohno and Miss N Ando for their excellent technical assistance and Dr Morris Ziff for helpful discussions.

1 Firestein G S, Zvaifler N J. Peripheral blood and synovial fluid monocyte activation in inflammatory arthritis. Arthritis Rheum 1987; 30: 857-63

2 Janossy G, Duke O, Poulter L W, Panayi G, Bofill M, Goldstein G. Rheumatoid arthritis: a disease of T-lymphocyte/macrophage immunoregulation. Lancet 1981 ; i: 839-42.

3 Ishikawa $\mathbf{H}$, Ziff $\mathbf{M}$. Electron microscopic studies of immunoreactive cells in the rheumatoid synovial membrane. Arthritis Rheum 1976; 19: 1-14.

4 Dayer J M, Stephenson M L, Schmidt E, Karge W, Krane S $M$. Purification of a factor from human blood monocytemacrophages which stimulates the production of collagenase macrophages which stimulates the production of collagenase and prostaglandin $E_{2}$ by cells cultured from

5 Schnyder J, Payne T, Dinarello C A. Human monocyte or recombinant interleukin l's are specific for the secretion of a metallo-proteinase from chondrocytes. I Immunol 1987; 138: 496-503.

6 Clarris J R E, Hamilton J A. Peripheral blood mononuclear cells stimulate $\mathrm{N}$-acetyl- $\beta$-glucosaminidase levels in human synovial fibroblast-like cells. Rheumatol Int 1985; 5: 55-60.

7 Matsubara T, Ziff $M$. Increased superoxide anion release from human endothelial cells in response to cytokines. $\mathcal{F}$ Immunol 1986; 137: 3295-8.

8 Hamilton J A, Leizer T, Ligelbach S R. The stimulation of human synovial fibroblast plasminogen activator activity: involvement of cyclic AMP and cyclooxygenase products. Biochim Biophys Acta 1986; 886: 195-202.

9 Mochan E, Uhl J, Newton R. Evidence that interleukin 1 induction of synovial cell plasminogen activator is mediated via prostaglandin $\mathrm{E}_{2}$ and cAMP. Arthritis Rheum 1986; 29: 1078-84.

10 Arend W P, Joslin F G, Massoni R J. Effects of immune complexes on production by human monocytes of interleukin 1 or an interleukin 1 inhibitor. $F$ Immunol 1985; 134: 3868-75.

11 Stimpson S A, Dalldorf F G, Otterness I G, Schwab J H. Exacerbation of arthritis by IL-1 in rat joints previously injured by peptidoglycan-polysaccharide. F Immunol 1988; 140: 2964-9.

12 Ratcliffe A, Tyler J A, Hardingham T E. Articular cartilage cultured with interleukin 1 . Increased release of link protein, hyaluronate-binding region and other proteoglycan fragments. Biochem $\mathcal{F}$ 1986; 238: 571-80.

13 Pohlman T H, Stanness K A, Beatty P G, Ochs H D, Harlan $J \mathbf{M}$. An endothelial cell surface factor(s) induced in vitro by lipopolysaccharide, interleukin 1 , and tumor necrosis factor- $\alpha$ increases neutrophil adherence by a CDw18 dependent mechanism. I Immunol 1986; 136: 4548-53.

14 Cavender D E, Haskard D O, Joseph B, Ziff M. Interleukin 1 increases the binding of human $B$ and $T$ lymphocytes to
endothelial cell monolayers. F Immunol 1986; 136: 203-7.

15 Claris B J, Fraser J R E, Ash P, Leiser T, Hamilton J A Interleukin- $1 \beta$ and interleukin- $\alpha$ stimulate the $\mathrm{N}$-acetyl- $\beta$ Interleukin- $1 \beta$ and interleukin- $1 \alpha$ stimulate the $N$-acetyl- $\beta$ -
glucosaminidase activity of human synovial cells. Rheumatol Int 1987; 7: 271-5.

16 MacKinnon S K, Starkebaum G. Monocyte Fc receptor function in rheumatoid arthritis. Enhanced cell-binding of IgG induced by rheumatoid factors. Arthritis Rheum 1987 30: 498-506.

17 Kay N E, Douglas S D. Monocyte metabolic activation in patients with rheumatoid arthritis (40541). Proc Exp Bio Med 1979; 161: 303-6.

18 Seiz $M$, Hunstein $W$. Enhanced prostaglandin release from monocytes of patients with rheumatoid arthritis and systemic lupus erythematosus. Ann Rheum Dis 1985; 44: 438-45.

19 Danis V A, March L M, Nelson D S, Brooks P M Interleukin-1 secretion by peripheral blood monocytes and synovial macrophages from patients with rheumatoid arthritis. F Rheumatol 1987; 14: 33-9.

20 Shore A, Jaglal S, Keystone E C. Enhanced interleukin generation by monocytes in vitro is temporally linked to an early event in the onset or exacerbation of rheumatoid arthritis. Clin Exp Immunol 1986; 65: 293-302. 
21 Ropes M W, Bennett G A, Cobb S, Jacox R, Jessar R A. 1958 revision of diagnostic criteria for rheumatoid arthritis. Bull Rheum Dis 1958; 9: 175-6.

22 Steinbrocker $\mathrm{O}$, Traeger $\mathrm{C} \mathrm{H}$, Batterman R C. Therapeutic criteria in rheumatoid arthritis. $7 A M A$ 1949; 140: 659-62.

23 Lansbury J. Quantitation of activity of rheumatoid arthritis: method for summation of systemic indices of rheumatoid activity. Am ₹ Med Sci 1956; 232: 300-17.

24 Toyoda $\mathbf{K}$, Saito S, Naito S, et al. HLA antigens in classical and malignant rheumatoid arthritis in Japanese population. Tissue Antigens 1977; 10: 56-9.

25 Kent J F, Fife E H. Precise standardization of reagents for complement fixation. Am F Trop Med 1963; 12: 103-16.

26 LeMaeshall J, Fraser J R E, Muirden K D. Lysosomal activation by neutral saccharides in cell cultures of synovium. activation by neutral saccharides in

27 Pick E, Mizel D. Rapid microassays for the measurement of superoxide and hydrogen peroxide production by macrophages in culture using an automatic enzyme immunoassay reader. F Immunol Methods 1981; 46: 211-26.

28 Van Geider B F, Slater E C. The extinction coefficient of cytochrome C. Biochim Biophys Acta 1962; 58: 593-5

29 Tanaka K, Ishikawa E, Ohmoto Y, Hirai Y. In vitro production of human interleukin $l \alpha$ and interleukin $1 \beta$ by peripheral blood mononuclear cells examined by sensitive sandwich enzyme immunoassay. Eur $\mathcal{F}$ Immunol 1987; 17: 1527-30.

30 Nishida T, Nishino N, Takano M, et al. cDNA clotting of IL-1 1 and IL-1B from mRNA of U937 cell line. Biochem IL-lu and IL-1/ from mRNA of U937

31 Hirai Y, Masui Y, Nakai S, Kikumoto Y, Hong Y-M. Interleukin-1: cDNA clotting, production and biological activities of human interleukin-1. Gann monograph on cancer research: cellular and molecular mechanism of tumor immunity. Tokyo: Japan Scientific Societies Press, 1988: 156-66.

32 Dominguez J H, Mundy G R. Monocytes mediate osteoclastic bone resorption by prostaglandins production. Calcif Tissue Int 1980; 31: 29-34.

33 Burmester G R, Dimitriu-Bona A, Waters S J, Winchester $R$ J. Identification of three major synovial lining cell populations by monoclonal antibodies directed to la antigens and tions by monoclonal antibodies directed to la antigens and antigens associated with monocyte/macroph

34 Klickstein L B, Shapleigh C, Goetzl E. Lipooxygenation of arachidonic acid as a source of polymorphonuclear leukocyte chemotactic factors in synovial fluid and tissue in rheumatoid arthritis. $\mathcal{F}$ Clin Invest 1980; 66: 1166-70.

35 Whicher J T, Gilbert A M, Westacott C, Hutton C. Defective production of leucocyte mediator (interleukin 1) by peripheral blood leucocytes of patients with systemic sclerosis, systemic lupus erythematosus, rheumatoid arthritis and mixed connective tissue disease. Clin Exp Immunol 1986; 65: 80-9.

36 Muirden $\mathrm{K}$ D. Lysosomal enzymes in synovial membrane in rheumatoid arthritis. Ann Rheum Dis 1972; 31: 265-71.

37 Wegelis O, Klockars M, Vaino U. Acid phosphatase activity in rheumatoid synovium. Acta Med Scand 1968; 183: $549-51$.

38 Dingle J T. The secretion of enzymes into the pericellular environment. Philos Trans R Soc Lond [Biol] 1975; 271: 315-24.

39 Eeckhout Y, Vaes G. Further studies on the activation of procollagenase, the latent precursor of bone collagenase. Effects of lysosomal cathepsin B, plasma and kallikrein, and spontaneous activation. Biochem $\mathcal{f} 1977 ; 166: 21-31$.

40 Hurst N P, Bessac B, Nuki G. Monocyte superoxide anion production in rheumatoid arthritis: preliminary evidence for enhanced rates of superoxide anion production by monocytes from patients receiving penicillamine, sodium aurothiomalate and corticosteroids. Ann Rheum Dis 1984; 43: 28-33.

41 Nakagawara A, Nathan C F, Cohn Z A. Hydrogen peroxide metabolism in human monocytes during differentiation in vitro. $\mathcal{F}$ Clin Invest 1981 ; 68: 1243-52.

42 French J K, Hurst N P, McColl S R, Cleland L. Effects of piroxicam on superoxide generation, phospholipid methylapiroxicam on superoxide generation, phospholipid methylanuclear cells. $\mathcal{F}$ R heumatol 1987; 14: 1018-21.

43 Hurst N P, Bell A L, Nuki G. Studies of the effect of Dpenicillamine and sodium aurothiomalate therapy on superoxide anion production by monocytes from patients with rheumatoid arthritis: evidence for in vivo stimulation of monocytes. Ann Rheum Dis 1986; 45: 37-43.

44 Haynes D R, Garrett I R, Whitehouse M W, Vernon-Roberts B. Do gold drugs inhibit interleukin-1? Evidence from an in vitro lymphocyte activating factor assay. $\mathcal{F}$ R heumatol 1988 15: 775-8.

45 Kern J A, Lamb R J, Reed J C, Daniele R P, Nowell P C. Dexamethasone inhibition of interleukin 1 beta production by human monocytes. $\mathcal{f}$ Clin Invest 1988; 81: 237-44. 